\title{
Center of Gravity Based, Switching and Partial Modeled Gait Planning
}

\author{
Sabri Yilmaz and Metin Gokasanand Seta Bogosyan
}

\begin{abstract}
This paper aims for a new structure that can achieve walking by kinematic solutions. The main contribution is having kinematic model of a biped and makes it to have step on open loop form. The paper takes the 5-DOF leg, uses Denavit-Hartenberg convention to obtain forward kinematic models, uses Jacobian matrix to obtain inverse kinematic models. Uses a switching mode models, and switching gaits for tip points and center of gravity. The models and gaits switch by changing support and swing foots. Models are divided into four different models and when models switch two models become active. Using two models gives chance to define two paths for biped, for one step and center of gravity can be controlled separately. By these solutions study aims to make biped to follow these paths. The simulation results from Matlab proved that tip points and center of gravity can make the aimed walking, so that by kinematic constraints stable dynamic walking can be achieved.
\end{abstract}

Index Terms-Biped robot, forward kinematics, gait planning.

\section{INTRODUCTION}

Biped walking is one of the most exciting topics in robotics. In general, the main purpose is to get stable walking dynamics after that stable running dynamics are worked.

Working procedure develops step by step. First of all defining structure and deriving kinematic model is done. The existing studies use different models and different amount of DOF. For example leg can be modeled by 3-DOF, each joint consists of only pitch movement [1]. Some other works model the biped leg by 5-DOF, hip joint consists of roll and pitch axis, knee joint consists of only pitch axis and ankle joint consists of roll and pitch axis like hip joint [2]. And the other works model the leg by 6-DOF, hip joint consists of roll, pitch and yaw axis, knee joint and ankle joint have same axis like 5-DOF model [3]. In this paper leg modeled by 5 -DOF, not using yaw axis in will make model simpler. This study aims walking across linear path so yaw axis has no effect on walking. After defining structure, kinematic model was derived. Denavit-Hartenberg convention used for kinematic model [4]. From the homogenous transformation matrices found by Denavit-Hartenberg convention inverse kinematics found by Jacobian method [5]. Legs were modeled together, but each leg has two different Jacobian matrixes. One is for the leg's support position, other one is for swing position. When right leg is the support leg, the base frame of Jacobian matrix will be on right foot, and the first tip will be on center of gravity. Second Jacobian matrix will take

Manuscript received May 15, 2013; revised July 20, 2013.

The authors are with the Istanbul Technical University Electrical and Electronics Faculty, Maslak, 34469 Istanbul Turkey (e-mail: yilmazsabr@itu.edu.tr; gokasan@itu.edu.tr; sbogosyan@alaska.edu). center of gravity as the base frame and will take left foot as tip point. At this configuration again one part of the partial function will be used as path [6].

After defining kinematic side of the walking, dynamic modeling is derived. There are two widely used models available. Zero-Moment Point (ZMP) and Inverted Pendulum (IP) [7]-[9]. ZMP is based on controlling the motion of the ZMP where movement and body dynamics do not cause any moments. IP uses pendulum model and controls the leg as a pendulum. In this study, instead of these models another path is defined for center of gravity, where is accepted between two legs. The path that is defined for center of gravity is another half circular path but width and period of this sinusoidal function is different from path that is defined for legs.

When support leg and swing leg change the kinematic models change. Biped modeled by four different models and every configuration has two models. By this division biped uses two different kinematic models for one step. By using two different models, center of gravity and swing leg can be controlled separately. Support leg can be taken as a robot arm, and swing leg can be taken as robot arm that is on the moving platform. Defining sinusoidal paths makes solution very fast and simple. Controlling bipeds swing leg and center of gravity by simple sinusoidal functions gives chance to consider dynamic model by kinematic solution.

\section{KinEMATIC ANALYSIS}

In this section, the kinematic model will be derived of the leg system in consideration. To this aim, the derivation of the Denavit -Hartenberg parameters and Jacobian matrices will be discussed.

Biped walking can be modeled by a leg with six degrees of freedom. The three of DOF are for pitch axis; two of them are for roll axis and the last one for yaw axis. Walking can be modeled by pitch and the roll axis, so in this paper, the legs modeled by five degrees of freedom. This model is enough for aimed scenario, which aims for walking on a smooth surface. Center of gravity is accepted as positioning in the middle of two legs. Friction force between the surface and the robot foot is large enough for the legs avoid from slipping.

Kinematic modeling of a robot manipulator is based on the following homogeneous transformation [10].

$$
\begin{gathered}
H=\left[\begin{array}{cc}
R_{n}^{0} & o_{n}^{0} \\
0 & 1
\end{array}\right]=T_{n}^{0} \\
T_{n}^{0}=A_{1}\left(q_{1}\right) A_{2}\left(q_{2}\right) \ldots \ldots . A_{n}\left(q_{n}\right)
\end{gathered}
$$


where $R_{\mathrm{i}}^{0}$ is the rotation matrix, $o_{\mathrm{n}}^{0}$ is the translation vector, $A_{i}$ is the homogeneous transformation matrix and $q_{i}$ is the joint variable. $A_{i}$ is the function of joint variable $q_{i}$, gives the position and the orientation of the frame with respect to the previous frame. $T_{\mathrm{n}}^{0}$ gives the position and the orientation of the tool frame which is the last frame with respect to the initial frame.

In this paper, Denavit-Hartenberg convention used to derive kinematic model of the manipulator. With the use of this convention, the position and orientation of a body can be given as the product of four homogeneous transformations. [10].

$$
A_{i}=\operatorname{Trans}_{z_{i-1}, d_{i}} \operatorname{Rot}_{z_{i-1}, \theta_{i}} \operatorname{Trans}_{x_{i}, a_{i}} \operatorname{Rot}_{x_{i}, a_{i}}
$$

where $\operatorname{Trans}_{z_{i-1}, d_{i}}$ is the translation from $z_{i-1}$ by the link offset $d_{i}, \operatorname{Rot}_{z_{i-1}, \theta_{i}}$ is the rotation around $z_{i-1}$ by the joint angle $\theta_{i}$, Trans $_{x_{i}, a_{i}}$ is the translation from $x_{i}$ by the link length $a_{i}$ and $\operatorname{Rot}_{x_{i}, \alpha_{i}}$ is the rotation around $x_{i}$ the link twist $\alpha_{i}$.

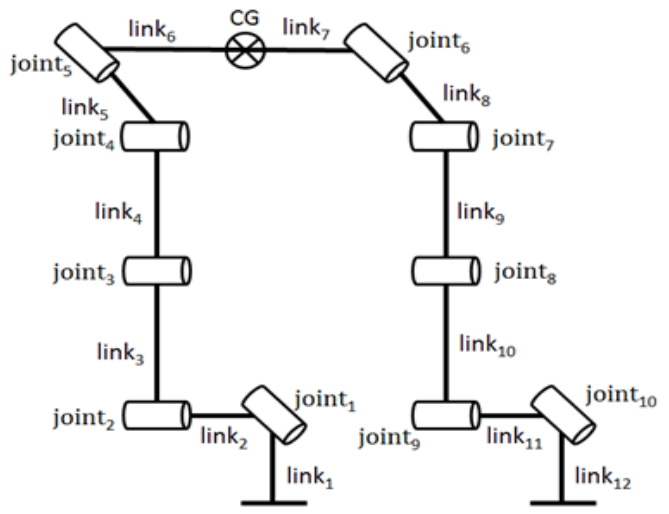

Fig. 1. Model used for legs with 5 DOF.

In Fig. 1 shows the stick model of the legs. The section from the link $k_{1}$ to the center of gravity $(C G)$ is named as the right leg (RL), while that from link 12 to $C G$ is named as the left leg (LL). The hip of the right leg consists of link ${ }_{5}$, joint 4 and joint ${ }_{5}$, while the knee of right leg consists of joint ${ }_{3}$ and ankle of right leg consists of link $_{2}$, joint jond joint $_{2}$. The LL parameters are similar to RL parameters. The hip consists of link $_{8}$, joint ${ }_{6}$ and joint $t_{7}$, the knee consists of joint ${ }_{8}$, while the ankle consists of link 11 , joint and joint $_{10}$. Link Lnd link $_{12}$ are the feet height, link $\mathrm{k}_{3}$ and link $\mathrm{k}_{10}$ are the shinbones, link $\mathrm{k}_{4}$ and link $_{9}$ are thighbones and link 6 and link ${ }_{7}$ are the hip offsets. This model is used for simplicity when defining Denavit-Hartenberg parameters. Hips and ankles are shown as linear manipulators, but the links creating these joint groups have zero length.

In this study four different Jacobian models used for inverse kinematics. Two of them are the inverse kinematics solution when the RL is the support leg, from RL to CG and from CG to LL. The other two for when the LL is the support leg, from LL to CG and from CG to RL. By this modeling manipulator, parts can be controlled separately. Fig. 2. shows the coordinate frames are put with respect to Denavit-Hartenberg convention and right hand rule on the model from right to left leg.

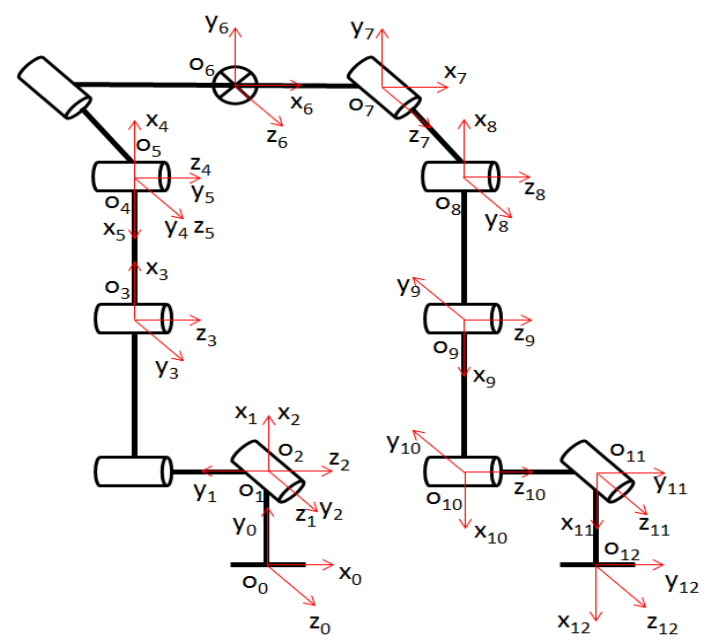

Fig. 2. Coordinate frames from R to LL.

The model in Fig. 2 used for finding Jacobian matrices from right to left leg, these matrices are used when right foot is the support foot and left foot is swing foot.

TABLE I: DENAVIT-HARTENBERG PARAMETERS F
\begin{tabular}{|l|l|l|l|l|}
\hline \multirow{2}{*}{$\begin{array}{l}\text { Link } \\
\text { numbers }\end{array}$} & $\mathrm{a}$ & $\alpha$ & $\mathrm{d}$ & $\theta$ \\
\cline { 2 - 7 } & Parameters \\
\hline 1 & $\mathrm{~L}_{1}$ & 0 & 0 & $\pi / 2$ \\
\hline 2 & 0 & $\pi / 2$ & 0 & $\theta_{2}$ \\
\hline 3 & $\mathrm{~L}_{3}$ & 0 & $\mathrm{~L}_{2}$ & $\theta_{3}$ \\
\hline 4 & $\mathrm{~L}_{4}$ & 0 & 0 & $\theta_{4}$ \\
\hline 5 & 0 & $\pi / 2$ & 0 & $\theta_{5}$ \\
\hline 6 & $\mathrm{~L}_{6}$ & 0 & $\mathrm{~L}_{5}$ & $\theta_{6}$ \\
\hline 7 & $\mathrm{~L}_{7}$ & 0 & 0 & 0 \\
\hline 8 & 0 & $\pi / 2$ & $\mathrm{~L}_{8}$ & $\theta_{8}$ \\
\hline 9 & $\mathrm{~L}_{9}$ & 0 & 0 & $\theta_{9}$ \\
\hline 10 & $\mathrm{~L}_{10}$ & 0 & 0 & $\theta_{10}$ \\
\hline 11 & 0 & $\pi / 2$ & $\mathrm{~L}_{11}$ & $\theta_{11}$ \\
\hline 12 & $\mathrm{~L}_{12}$ & 0 & 0 & $\theta_{12}$ \\
\hline
\end{tabular}

Fig. 3 shows the coordinate frames from left to right leg. The base frames of Fig. 2 and Fig. 3 are chosen identical. This will brings simplicity when defining parameters and switching between legs.

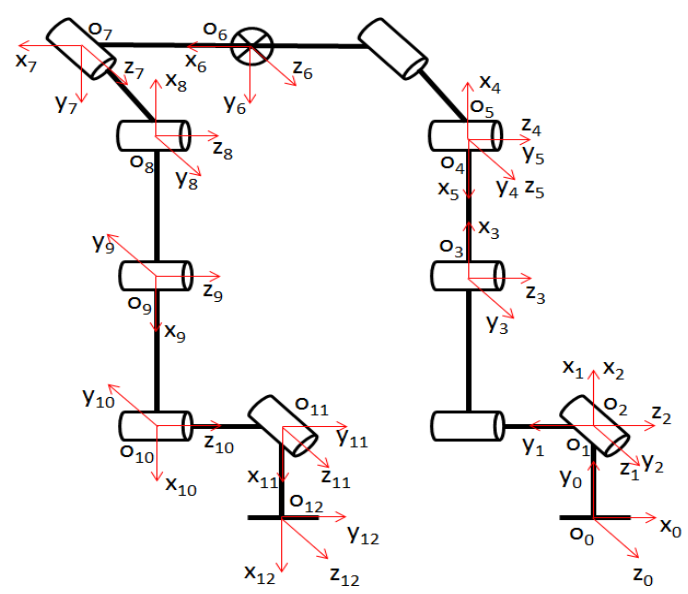

Fig. 2. Coordinate frames from $\mathrm{L}$ to $\mathrm{RL}$ 
TABLE II: DENAVIT-HARTENBERG PARAMETERS FOR L TO RL

\begin{tabular}{|l|l|l|l|l|}
\hline \multirow{2}{*}{$\begin{array}{l}\text { Link } \\
\text { numbers }\end{array}$} & \multicolumn{4}{|l|}{ Parameters } \\
\cline { 2 - 5 } 1 & $\mathrm{a}$ & $\alpha$ & $\mathrm{d}$ & $\theta$ \\
\hline 2 & $\mathrm{~L}_{1}$ & 0 & 0 & $\pi / 2$ \\
\hline 3 & 0 & $\pi / 2$ & 0 & $\theta_{2}$ \\
\hline 4 & $\mathrm{~L}_{3}$ & 0 & $\mathrm{~L}_{2}$ & $\theta_{3}$ \\
\hline 5 & $\mathrm{~L}_{4}$ & 0 & 0 & $\theta_{4}$ \\
\hline 6 & 0 & $\pi / 2$ & 0 & $\theta_{5}$ \\
\hline 7 & $\mathrm{~L}_{6}$ & 0 & $\mathrm{~L}_{5}$ & $\theta_{6}$ \\
\hline 8 & $\mathrm{~L}_{7}$ & 0 & 0 & 0 \\
\hline 9 & 0 & $\pi / 2$ & $\mathrm{~L}_{8}$ & $\theta_{8}$ \\
\hline 10 & $\mathrm{~L}_{9}$ & 0 & 0 & $\theta_{9}$ \\
\hline 11 & $\mathrm{~L}_{10}$ & 0 & 0 & $\theta_{10}$ \\
\hline 12 & 0 & $\pi / 2$ & $\mathrm{~L}_{11}$ & $\theta_{11}$ \\
\hline & $\mathrm{L}_{12}$ & 0 & 0 & $\theta_{12}$ \\
\hline
\end{tabular}

It can be noted that Table I and Table II are identical. The parameters in d column are equal to zero because they are the virtual lengths only defined to determine the coordinates frame for Denavit-Hartenberg convention. The a columns are identical, too, because both legs, hence their link lengths are identical. The joint angle $\theta_{12}$ is the angle of joint $t_{10}$ at the model from RL to LL; it's also the angle of joint ${ }_{1}$ at the model from LL to RL. The $\alpha$ columns are also identical as a result of the identical base frames.

Jacobian matrices are created by using homogeneous transformation matrices.

$$
\begin{gathered}
v_{n}^{0}=J_{v} \dot{q} \\
v_{n}^{0}=\left[\begin{array}{c}
v_{n_{x}}^{0} \\
v_{n_{y}}^{0} \\
v_{n_{z}}^{0}
\end{array}\right]
\end{gathered}
$$

$v_{n}^{0}$ is the linear velocity vector of the tip point, $\dot{q}$ is the velocity matrix of the joints and $J_{v}$ is the linear part of the Jacobian matrix.

$$
\begin{gathered}
w_{n}^{0}=J_{w} \dot{q} \\
w_{n}^{0}=\left[\begin{array}{c}
w_{n_{x}}^{0} \\
w_{n_{y}}^{0} \\
w_{n_{z}}^{0}
\end{array}\right]
\end{gathered}
$$

$w_{n}^{0}$ is the angular velocity vector of the tip point and $J_{w}$ is the angular part of the Jacobian matrix.

$$
\begin{gathered}
\xi=J \dot{q} \\
\xi=\left[\begin{array}{l}
v_{n}^{0} \\
w_{n}^{0}
\end{array}\right]
\end{gathered}
$$

$\xi$ is the velocity matrix of the tip point, combining of linear and angular velocity vectors of the tip point.

$$
J=\left[\begin{array}{l}
J_{v} \\
J_{w}
\end{array}\right]
$$

$J$ is the Jacobian matrix.

$$
\begin{aligned}
& J_{v_{i}}= \begin{cases}z_{i-1} \times\left(o_{n}-o_{n-1}\right) & \text { for i revolute } \\
z_{i-1} & \text { for i prismatic }\end{cases} \\
& J_{v_{i}}= \begin{cases}z_{i-1} & \text { for i revolute } \\
0 & \text { for i prismatic }\end{cases}
\end{aligned}
$$

$z_{i}$ is the rotation vector about $\mathrm{z}$ axis found by the homogenous transformation matrix, $o_{i}$ is the translation vector found by the homogenous transformation matrix, too.

$$
\dot{\theta}=J^{-1} \xi
$$

Matrix product of inverse of the Jacobian matrix and the velocity matrix $\xi$ will result in the velocity $\dot{\theta}$ of the joints.

In this paper, only the linear velocity of the tip point is studied. So the velocity matrix of the tip point loses dimension and becomes linear velocity vector $v_{n}^{0}$. By this operation Jacobian matrix again loses the dimension and consists of only $J_{v}$ parameters. Because of having five degrees of freedom, Jacobian matrix' dimension becomes $3 \times 5$, it is not a square matrix, so pseudo inverse is used. Inverse of the Jacobian matrix' dimension is $5 \times 3$, so the result of the product is $\dot{\theta}$ with the dimension of $5 \times 1$, angular velocities of the joints.

The four Jacobian matrices are defined as follows:

When RL is the support leg, $J_{1}$ and $J_{2}$ activated. $J_{1}$ is the Jacobian matrix from base of the right leg to center of gravity; $J_{2}$ is from center of gravity to base of the LL.

When LL is the support leg, $J_{3}$ and $J_{4}$ activated. $J_{3}$ is the Jacobian matrix from base of the left leg to center of gravity; $J_{4}$ is from center of gravity to base of the RL.

\section{GAIT PlanNING}

In this section, gait planning is studied. Half circular paths will be defined for biped; they will be created from partial functions for RL, LL and CG.

In this paper sinusoidal paths are defined for gait planning and walking cycle is divided into three different parts, first step, alternating steps and last step. When RL is the support leg first model is activated and two different paths are used in inverse kinematics solutions. One is for $J_{1}$, the other one is for $J_{2}$. When LL is the support leg second model is activated, similarly one path is for $J_{3}$ and the other path for $J_{4}$.

Walking steps:

LL is defined as support leg, $J_{3}$ and $J_{4}$ activate.

When center of gravity's projection is in the middle of two legs, $J_{1}$ and $J_{3}$ activate, two legs are the support legs.

When center of gravity's projection is on the right foot, $J_{1}$ and $J_{2}$ activate, RL is the support leg.

When center of gravity's projection is in the middle of two 
legs, $J_{1}$ and $J_{3}$ activate, two legs are the support legs.

Again, three steps are repeated alternately.

To obtain curve for the path of the center of gravity partial functions are created.

$$
\text { First step: }\left\{\begin{array}{l}
X_{c g}=0.075 \cos \left(\frac{2 \pi t}{0.6}\right) \\
Z_{c g}=0.03 \cos \left(\frac{2 \pi t}{0.6}\right)
\end{array}(0 \leq \mathrm{t} \leq 0.3 \mathrm{~s})\right.
$$

Alternating steps:

$$
\begin{aligned}
& \left\{\begin{array}{l}
X_{c g}=0.075 \cos \left(\frac{\pi(t-0.3)}{0.6}\right) \\
Z_{c g}=0.06 \sin \left(\frac{\pi(t-0.3)}{0.6}\right) \\
\text { Last step: }
\end{array}\right. \\
& \left\{\begin{array}{l}
\left.X_{c g}=0.3 \leq \mathrm{t} \leq 0.9 \mathrm{~s}\right) \\
Z_{c g}=0.06 \sin \left(\frac{2 \pi(t-0.3)}{0.6}\right)
\end{array}(0.9 \leq \mathrm{t} \leq 1.2 \mathrm{~s})\right.
\end{aligned}
$$

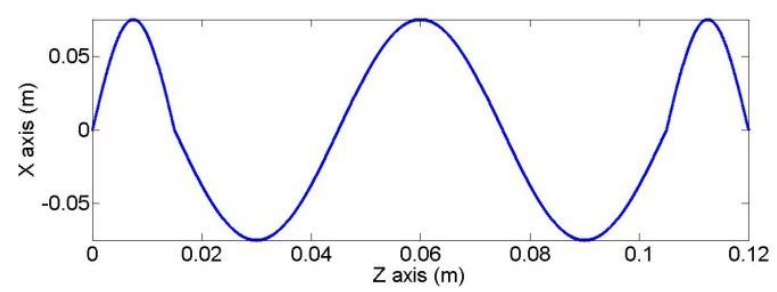

Fig. 4. Defined path for CG

Fig. 4 shows the defined path for the center of gravity, $z$ axis is the walking direction, $x$ axis lateral movement direction. First step and the last steps length are half of the alternating steps.To obtain curve for the path of the legs partial functions are created.

First step: $\left\{\begin{array}{l}Y_{c g}=0.06 \times \cos \left(\frac{4 \pi t}{0.6}\right) \\ Z_{c g}=0.03 \times \sin \left(\frac{4 \pi t}{0.6}\right)\end{array}(0 \leq \mathrm{t} \leq 0.15 \mathrm{~s})\right.$

Alternating steps:

$$
\begin{aligned}
& \text { For LL: }\left\{\begin{array}{l}
Y_{c g}=0.075 \times \cos \left(\frac{2 \pi(t-0.15)}{0.6}\right) \\
Z_{c g}=0.06 \times \sin \left(\frac{2 \pi(t-0.15)}{0.6}\right)
\end{array}(0.15 \leq \mathrm{t} \leq 0.45 \mathrm{~s})\right. \\
& \text { For RL: }\left\{\begin{array}{l}
Y_{c g}=0.075 \times \cos \left(\frac{2 \pi(t+0.15)}{0.6}\right) \\
Z_{c g}=0.06 \times \sin \left(\frac{2 \pi(t+0.15)}{0.6}\right)
\end{array}(0.45 \leq \mathrm{t} \leq 0.75 \mathrm{~s})\right. \\
& \text { For LL: }\left\{\begin{array}{l}
Y_{c g}=0.075 \times \cos \left(\frac{2 \pi(t-0.3)}{0.6}\right) \\
Z_{c g}=0.06 \times \sin \left(\frac{2 \pi(t-0.3)}{0.6}\right)
\end{array}(0.75 \leq \mathrm{t} \leq 1.05 \mathrm{~s})\right.
\end{aligned}
$$

$$
\text { Last step: }\left\{\begin{array}{l}
Y_{c g}=0.06 \times \cos \left(\frac{4 \pi(t-0.15)}{0.6}\right) \\
Z_{c g}=0.03 \times \sin \left(\frac{4 \pi(t-0.15)}{0.6}\right)
\end{array}(1.05 \leq \mathrm{t} \leq 1.2 \mathrm{~s})\right.
$$

RL starts walking and stops walking, RL only takes alternating steps. After first step ends at $\mathrm{t}=0.15 \mathrm{~s}$, alternating steps start and by the period $\mathrm{T}=0.3 \mathrm{~s}$ partial functions change for the right and the left leg. After alternating steps are taken, right leg takes last step.

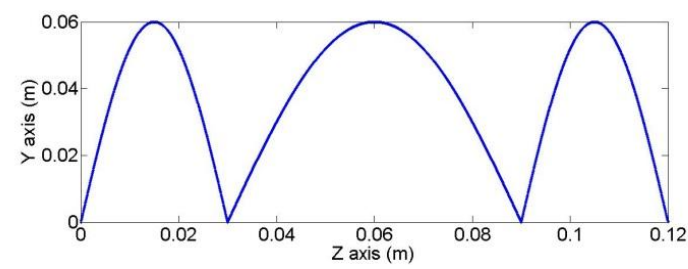

Fig. 5. Defined path for RL.

In Fig. 5 right leg takes first step, a full step and the last step. The first and the last steps are half of the length of the alternating steps. The alternating step length is $0.06 \mathrm{~m}$. Fig. 6 . shows elevation of the right leg vs. time.

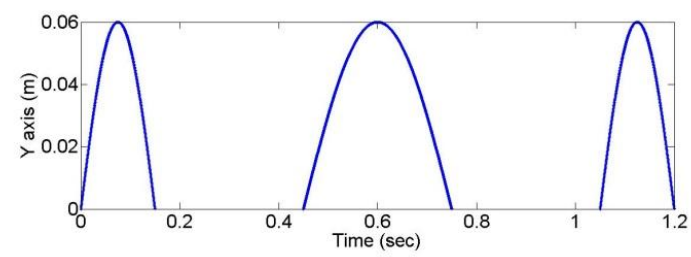

Fig. 6. RL's path on time axis.

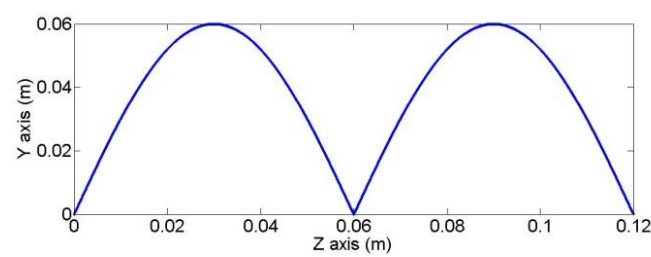

Fig. 7. Defined path for LL.

In Fig. 7, left leg takes two alternating steps. In $\mathrm{z}$ axis center of gravity, right leg and left leg complete movement of $0.12 \mathrm{~m}$. Fig. 8 shows elevation of the left leg vs. time.

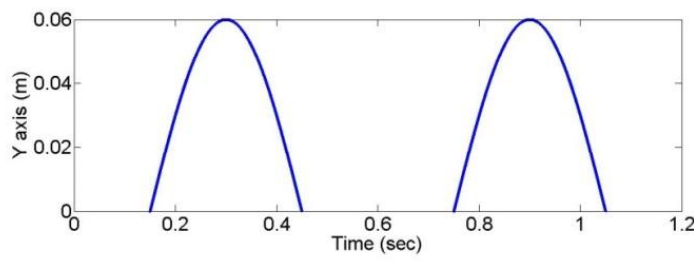

Fig. 8. LL's path on time axis

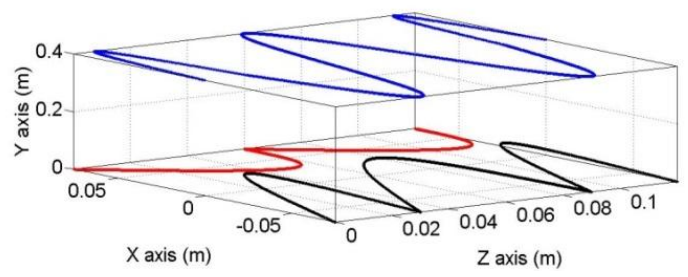

Fig. 9. Defined paths for CG (blue), RL (black) and LL (red) together 
Upon Fig. 9. shows the generated sinusoidal functions on $\mathrm{x}-\mathrm{y}-\mathrm{z}$ axis. If the figure examined, first RL has first step, while CG moves upon LL. After RL reaches surface, again CG comes between two legs, LL starts moving across its defined path, the walking motion goes on like this switching between functions and legs.

\section{Simulation RESUlts}

The proposed models and gait are tested in this section. Asdeclared in Section II gait is generated only for walking. In Fig. 10 movement of the biped is shown.

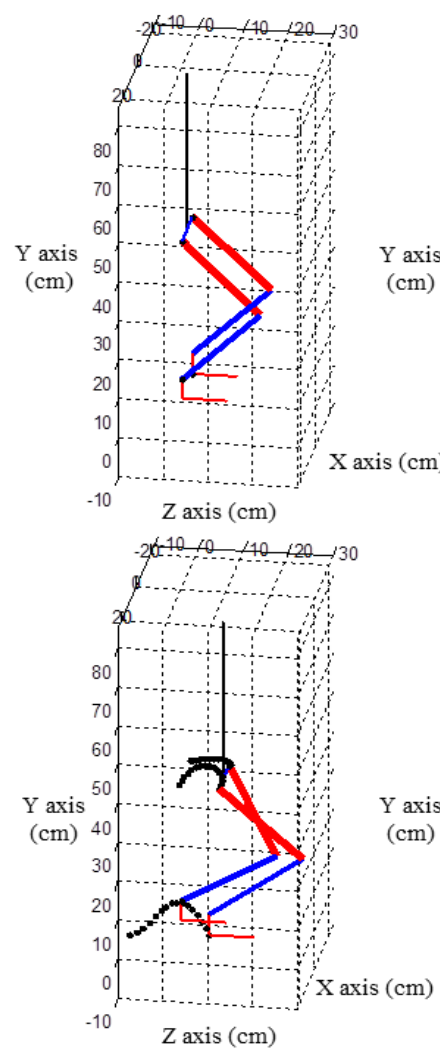

$\mathrm{Z}$ axis $(\mathrm{cm})$
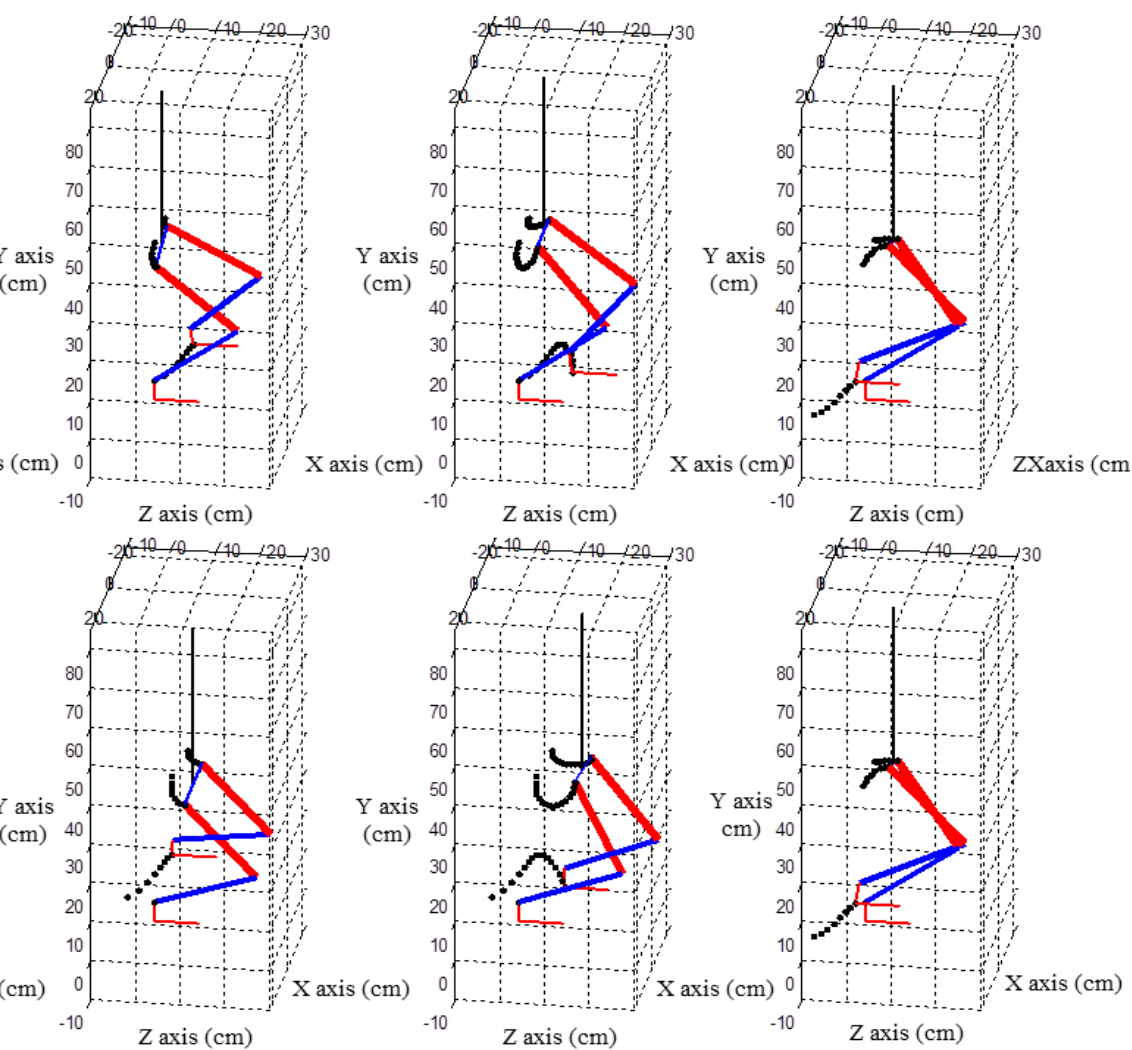

Fig. 10. Movement of the biped.

The upper red lines in Fig. 10. show the thighbones, under of them blue lines show the shinbones. Under shinbones the "L" shaped red lines show the feet and between the shinbones black line shows the hip and on of the hip vertical line shows the body of biped. Fig. 10. a shows the bipeds position ready for walking, the biped's legs are not positioned vertically because it will cause solution to fail because Jacobian matrix' rank will lose dimension. Fig. 10. b and c shows the complete first step of the biped. With Fig. 10. d alternating steps begin. From this figure it is shown that by this gait planning biped walks stable, CG can be controlled as it is desired.

\section{CONCLUSIONS}

Forward kinematic models of a biped was obtained from Denavit-Hartenberg convention. From these models inverse kinematic models were obtained. For these inverse kinematic models, basic half circular paths defined, aiming to walk in one direction on the smooth surface. These paths have derived in time and by using tip point velocities and Jacobian matrices, joint velocities are obtained. Manipulators followed the paths by some steady-state error because of being open loop systems.

In Simulation Results Section, the movement figures of the biped are shown, it is clearly seen without any controller the biped will not follow the desired path. Designing propercontrollers for the manipulators will minimize or get rid of the errors and biped can walk smoothly as planned.

\section{REFERENCES}

[1] Y. Huang, B. Vanderborght, R. V. Ham, M. V. Damme, G. Xie, and D Lefeber, "Step Length And Velocity Control Of A Dynamic Bipedal Walking Robot With Adaptable Compliand Joints," IEEE/ASME Transactions on Mechatronics, vol. 18, pp. 598-611, Apr. 2013.

[2] N. T. Phuong, D. W. Kim, H. K. Kim, and S. B. Kim, "An Optimal Control Method For Biped Robot With Stable Walking Gait," in Proc. IEEE-Ras International Converence on Humanoid Robots, pp. 211-218, Dec. 2008.

[3] J. L. Yan and H. P. Huang, "A Fast And Smooth Walking Pattern Generator Of Biped Robot Using Jacobian Inverse Kinematics," in Proc. IEEE Workshop on Advanced Robotics and Its Social Impacts, 9-11 Dec. 2007, pp. 1-6.

[4] M. Tarokh and M. Lee, "Kinematics Modeling Of Multi-Legged Robots Walking On Rough Terrain," in Proc. Second International Conference on Future Generation Communication and Networking Symposia, 13-15 Dec. 2008, vol. 4, pp. 12-16.

[5] D. Wholherr and M. Buss, "Posture Modification for Biped Humanoid Robots based on Jacobian Method," in Proc. IEEE/RSJ International Conference on Intelligent Robots and Systems, pp. 124-129, 2004.

[6] Q. Zhang, D. Chen, and H. Li, "A Gait planning method for biped Heel-And-Toe robot," in Proc. International Conference on Future Energy, Environment, and Materials, pp. 1799-1805, 2012.

[7] M. Vukobratovic and B. Borovac, "Zero-moment point - thirty five years of its life," International Journal of Humanoid Robotics, pp. 157-173, 2004.

[8] Y. Choi, D. Kim, and B. J. You, "On the walking control for humanoid robot based on the Kinematic resolution Of Com Jacobian with 
embedded motion," in Proc. IEEE International Conference on Robotics and Automation, pp. 2655-2660, May 2006.

[9] Z. Tang and M. J. Er, "Humanoid 3D Gait Generation based on inverted pendulum model," in Proc. 22nd IEEE International Symposium on Intelligent Control Part of IEEE Multi-conference on Systems and Control, pp. 339-344, Oct. 2007.

[10] J. J. Craig, Introduction to Robotics: Mechanics and Control, Third Edition, Prentice Hall.

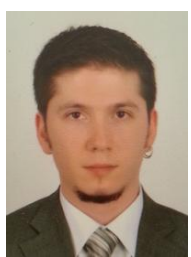

Sabri Yilmaz was born in Samsun, 1988. He has received his B.Sc. degrees in control engineering and mechanical engineering from Istanbul Technical University in 2011, 2013, respectively. He is currently a research assistant at the Control Engineering Department of Istanbul Technical University.

His research areas are robot kinematics and dynamics, humanoid walking, microcontroller based control systems and control of mechatronic systems.

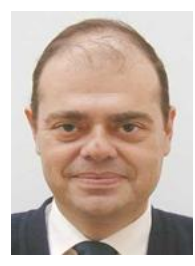

Metin Gokasan was born in Istanbul, 1958. He has received his B.Sc., M.Sc. and Ph.D. from Istanbul Technical University, Turkey in electrical and control engineering in 1980, 1982 and 1990, respectively. He is currently Professor and Department Chair at the Control
Engineering Department of Istanbul Technical University. He also worked as a visiting scholar between 2003 and 2006 at the University of Alaska Fairbanks.

His research interests are control of electrical machinery, power electronics and electrical drives, control of hybrid electric vehicles, mechatronics systems, and wind energy systems. He has authored 2 books on electrical machinery and power electronics, and over 80 journal and conference publications.

$\mathrm{He}$ is consultant to numerous transportation and energy companiesin Turkey.

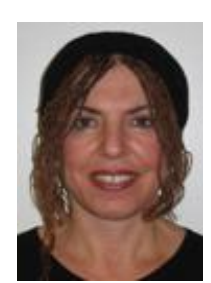

Seta Bogosyan was born in Istanbul, 1955. He has received her B.Sc. and M.Sc. degrees in electrical and control engineering from Istanbul Technical University (ITU)in 1981, 1983, respectively. She has conducted her $\mathrm{PhD}$ work jointly in University of California at Santa Barbara and ITU, and received her degree in 1991. She is currently a professor of Electrical and Computer engineering Department at University of Alaska Fairbanks

Her research areas are motion control, high efficiency control of hybrid electric vehicles, teleoperation/bilateral control systems, and applications of nonlinear control/estimation techniques to electromechanical and renewable energy systems. She has authored over a hundred journal and conference proceedings and several books. She has also served as associate editor in several IEEE transactions and Autosoft. 